\title{
Anomaly in the relaxation dynamics close to the surface plasmon resonance
}

\author{
Guillaume Weick ${ }^{1,2,3}$, Dietmar Weinmann ${ }^{1}$, Gert-Ludwig Ingold ${ }^{2}$ and Rodolfo A. Jalabert ${ }^{1}$ \\ 1 Institut de Physique et Chimie des Matériaux de Strasbourg, UMR 7504 (CNRS-ULP), 23 rue du Loess, BP 43, \\ F-67034 Strasbourg Cedex 2, France \\ ' ${ }^{2}$ Institut für Physik, Universität Augsburg, Universitätsstraße 1, D-86135 Augsburg, Germany \\ 3 Fachbereich Physik, Freie Universität Berlin, Arnimallee 14, D-14195 Berlin, Germany
}

\begin{abstract}
PACS 78.67.-n - Optical properties of low-dimensional, mesoscopic, and nanoscale materials and structures

PACS 73.20.Mf - Collective excitations (including excitons, polarons, plasmons and other charge density excitations)

PACS 71.45.Gm - Exchange, correlation, dielectric and magnetic response functions, plasmons
\end{abstract}

\begin{abstract}
We propose an explanation for the anomalous behaviour observed in the relaxation dynamics of the differential optical transmission of noble-metal nanoparticles. Using the temperature dependences of the position and the width of the surface plasmon resonance, we obtain a strong frequency dependence in the time evolution of the transmission close to the resonance. In particular, our approach accounts for the slowdown found below the plasmon frequency. This interpretation is independent of the presence of a nearby interband transition which has been invoked previously. We therefore argue that the anomaly should also appear for alkaline nanoparticles.
\end{abstract}

Absorption and transmission of a laser beam by a metallic nanoparticle unveil the properties of its conduction electrons. The use of femtosecond lasers in pump-probe spectroscopy then gives access to the electron dynamics on extremely short time scales [1-6]. In particular, the time dependence of the transmission spectrum during and after the excitation by the pump laser allows to follow the details of the electron relaxation.

In pump-probe experiments, a pump laser with a wavelength much larger than the diameter of the nanoparticle ' couples to the centre of mass of the electron gas, thereby exciting the surface plasmon mode. The collective excitation decays on a very short timescale of the order of $10 \mathrm{fs}$. Subsequently, electron-electron interactions lead quite rapidly to the thermalisation of the electronic system at an elevated temperature. Only on longer timescales, of the order of $100 \mathrm{fs}$ up to picoseconds, the electron-phonon coupling leads to an equilibration of the electronic system with the lattice degrees of freedom. In the case of noblemetal nanoparticles, this picture has to be completed by the possibility of interband transitions which are absent in alkaline nanoparticles.

In ref. [2] the relaxation of the differential transmission spectrum of copper nanoparticles was found to exhibit a slowdown close to the plasmon frequency. This feature persists far beyond the lifetime of the plasmon excitation and therefore was attributed to the slowdown of the energy transfer from the electrons to the lattice for frequencies close to the plasmon resonance. These considerations lead to the following puzzle: How can the plasmon resonance frequency play a role for relaxation processes which occur on timescales much larger than the lifetime of this excitation?

The $s$ - $d$ multiband transition, which for copper has its onset close to the plasmon resonance, has been invoked as a possible explanation of the unexpected slowdown. A many-body effect based on the interband resonance scattering of $d$ holes with surface plasmons was proposed to lead to a strong frequency dependence of the relaxation [7].

Subsequent experiments with silver nanoparticles also found the slowdown in the transmission dynamics [4]. Firstly, these experiments demonstrated the generality of the effect. Secondly, they established that the slowdown is only found above an excitation threshold. Finally, because the interband transition in silver is far away from the plasmon frequency [6], the explanation put forward in refs. [7] does not apply.

In the present paper, we propose a generic explanation 
of the observed frequency dependence of the transmission dynamics which does not rely on interband transitions. Therefore it applies not only to copper, but also to silver and even alkaline nanoparticles. Our mechanism is based on recently derived results for the finite-temperature corrections to the width and the position of the plasmon absorption peak [8]. We show that the temperature dependencies of the two quantities lead to an anomaly in the evolution of the differential transmission spectrum with decreasing temperature. In particular, a slowdown in the relaxation of the optical transmission appears close to the plasmon resonance.

The quantity that is measured experimentally is the frequency-resolved time-dependent transmission $\mathcal{T}_{\text {on }}$ after the excitation of the system by a pump laser pulse. Then, the static equilibrium transmission $\mathcal{T}_{\text {off }}$ of the sample measured in the absence of the pump is subtracted to obtain the relative differential transmission $[2,6]$

$$
\frac{\Delta \mathcal{T}}{\mathcal{T}}=\frac{\mathcal{T}_{\text {on }}-\mathcal{T}_{\text {off }}}{\mathcal{T}_{\text {off }}}
$$

Experimentally, the observed exponential decay of $\Delta \mathcal{T} / \mathcal{T}$ with time allowed to extract a relaxation time which as a function of frequency presents the above-mentioned anomaly.

For the theoretical discussion we assume that after an initial transient time of about $100 \mathrm{fs}$ the electronic system is thermalised with a time-dependent temperature $T(t)$. In thermal equilibrium, the differential transmission can be related to the temperature-dependent absorption cross section $\sigma(\omega, T)$ by [9]

$$
\frac{\Delta \mathcal{T}}{\mathcal{T}}=-\frac{3}{2 \pi a^{2}}\left[\sigma(\omega, T)-\sigma\left(\omega, T_{0}\right)\right],
$$

where $T_{0}$ is the temperature of the nanoparticle before the excitation and $a$ is its radius. This relation between transmission and absorption holds when the reflectivity of the sample is negligibly small, as it is the case in typical experimental situations with a low density of nanoparticles embedded in a glassy matrix.

Assuming the Breit-Wigner form for the absorption cross section $\sigma$ of a nanoparticle in the frequency regime close to the plasmon resonance, we write

$$
\sigma(\omega, T)=s(a) \frac{\gamma(T) / 2}{\left[\omega-\omega_{\mathrm{sp}}(T)\right]^{2}+[\gamma(T) / 2]^{2}} .
$$

Here, $\omega_{\mathrm{sp}}(T)$ is the temperature-dependent resonance frequency of the surface plasmon excitation, $\gamma(T)$ is the temperature-dependent width of the resonance, and $s(a)$ is a normalisation prefactor which depends on the radius of the nanoparticle.

The knowledge of the temperature $T(t)$ as a function of time would give access to the time evolution of the differential transmission. In principle, $T(t)$ could be obtained for specific systems from a two-temperature model for the heat transfer from the electronic system to the lattice [10].
However, we choose not to follow this route since our explanation of the anomaly relies only on the fact that $T(t)$ is monotonically decreasing. The qualitative and generic features do not depend on the system parameters entering in the quantitative description but on the temperature dependencies of $\omega_{\text {sp }}$ and $\gamma$. For temperatures much smaller than the Fermi temperature $T_{\mathrm{F}}$ we can write

$$
\begin{gathered}
\omega_{\mathrm{sp}}(T)=\omega_{\mathrm{sp}}^{(0)}-\omega_{\mathrm{sp}}^{(2)}\left(\frac{T}{T_{\mathrm{F}}}\right)^{2}, \\
\gamma(T)=\gamma^{(0)}+\gamma^{(2)}\left(\frac{T}{T_{\mathrm{F}}}\right)^{2} .
\end{gathered}
$$

The dependence of the positive quantities $\omega_{\mathrm{sp}}^{(0)}, \gamma^{(0)}, \omega_{\mathrm{sp}}^{(2)}$ and $\gamma^{(2)}$ on the system parameters is specified below (eqs. (9)-(11)), but it is not needed for the following qualitative discussion.

Inserting the Breit-Wigner form (3) of the resonance into the expression (2) for the differential transmission, one can see that a temperature-dependent shift of the resonance frequency $\omega_{\mathrm{sp}}$ leads to an anomaly in the relaxation dynamics of the differential transmission. At a given temperature, $\Delta \mathcal{T} / \mathcal{T}$ results from the difference between two absorption curves which are shifted in frequency with respect to each other and have different widths. The frequency dependence of $\Delta \mathcal{T} / \mathcal{T}$ is obtained from eqs. (2)(5) and shown in fig. 1 for various temperatures. For a given temperature, there exist two frequencies $\omega_{1,2}^{\mathrm{c}}$ where the differential transmission vanishes. In the limit of small $T / T_{\mathrm{F}}$ these frequencies can be approximated by

$$
\begin{aligned}
\omega_{1,2}^{\mathrm{c}}(T) & =\omega_{\mathrm{sp}}^{(0)}+\omega_{\mathrm{sp}}^{(2)} \frac{\gamma^{(0)}}{\gamma^{(2)}} \\
& \times\left[1 \mp \sqrt{1+\left(\frac{\gamma^{(2)}}{2 \omega_{\mathrm{sp}}^{(2)}}\right)^{2}}\left(1+\frac{\gamma^{(2)}}{2 \gamma^{(0)}}\left(\frac{T}{T_{\mathrm{F}}}\right)^{2}\right)\right] .
\end{aligned}
$$

$\Delta \mathcal{T} / \mathcal{T}$ is negative for frequencies below $\omega_{1}^{\mathrm{c}}$ or above $\omega_{2}^{\mathrm{c}}$, and positive between these two frequencies. As the frequency $\omega_{1}^{\mathrm{c}}$ is very close to $\omega_{\mathrm{sp}}^{(0)}$, our assumption of a BreitWigner lineshape which is a good description close to the resonance does not represent an important restriction. However, this is not the case for $\omega_{2}^{\mathrm{c}}$, which is farther away from $\omega_{\mathrm{sp}}^{(0)}$. For the parameters chosen in fig. 1, $\omega_{2}^{\mathrm{c}}$ lies at the far right of the shown frequency interval. There, the detailed form of the absorption curve will be important. It might for example play a role whether one assumes the Breit-Wigner form (3) or the quasi-Lorentzian that has been used in ref. [6] to fit the plasmon resonance over a large frequency range. In addition, for the case of copper the effects occurring around $\omega_{2}^{\mathrm{c}}$ will be masked by the interband transition. Therefore we focus our discussion on the anomalous behaviour close to $\omega_{1}^{\mathrm{c}}$.

When the temperature of the electronic system decreases after the initial excitation, the position of the absorption peak moves back towards its low-temperature 


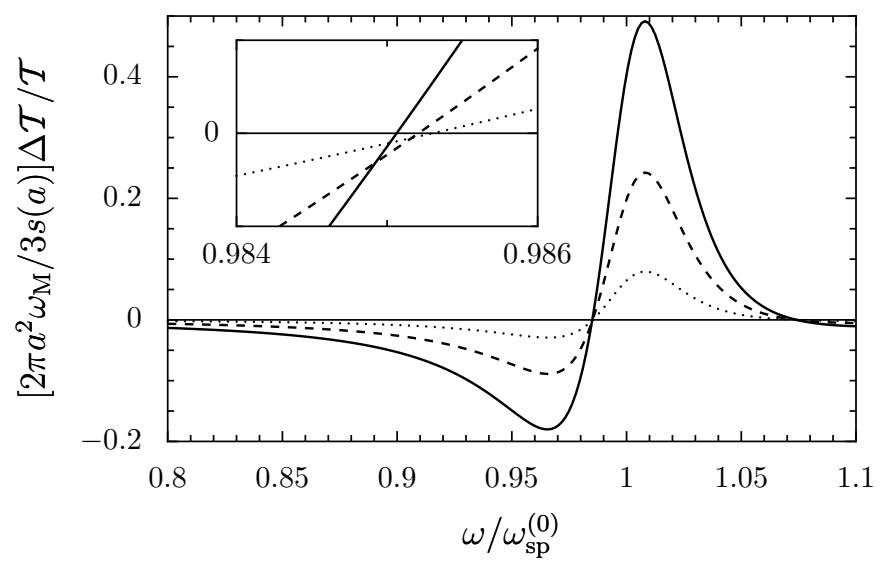

Fig. 1: The differential transmission for $\varepsilon_{\mathrm{F}} / \hbar \omega_{\mathrm{M}}=1, \varepsilon_{\mathrm{F}} / V_{0}=$ $0.5, k_{\mathrm{F}} a=15$, and $T_{0}=0$ (corresponding to a sodium nanoparticle with diameter $3.3 \mathrm{~nm}$ ) is shown as a function of the frequency $\omega$. The temperatures are $T / T_{\mathrm{F}}=0.05,0.035$, and 0.02 for the solid, dashed, and dotted line, respectively. The inset shows an enlargement of the region around $\omega_{1}^{\mathrm{c}}$ where $\Delta \mathcal{T} / \mathcal{T}$ changes sign.

frequency. During this relaxation process, the differential transmission is reduced in amplitude. The shift of $\omega_{1}^{\mathrm{c}}$ towards higher frequencies and the reduction in amplitude have opposite effects on the differential transmission for frequencies below $\omega_{1}^{\mathrm{c}}$. The contributions subtract and lead to a slowdown of the relaxation. As an example, one may consider the crossing of the solid and the dashed line in the inset of fig. 1. Here, the differential transmission at $T / T_{\mathrm{F}}=0.035$ (dashed line) has the same value as at the higher temperature 0.05 (solid line). In contrast, for frequencies slightly above $\omega_{1}^{\mathrm{c}}$, the two effects add up and the relaxation is accelerated. A very special dynamics can occur at frequencies which are crossed by $\omega_{1}^{\mathrm{c}}$ during the temperature relaxation. In such a situation, the differential transmission goes through zero and changes sign as the temperature decreases (see the inset in fig. 1).

For the example of alkaline nanoparticles, where only $s$ conduction electrons are relevant and no interband transition is present in the frequency regime of the plasmon resonance, we can show in detail how the mechanism presented above leads to the anomaly in the transmission relaxation. For this purpose, we use the temperature dependencies of the resonance frequency and of its width, which have recently been calculated [8] for temperatures well below $T_{\mathrm{F}}$.

In the approach of refs. [8] and [11], one decomposes the Hamiltonian $H=H_{\mathrm{cm}}+H_{\text {rel }}+H_{\mathrm{c}}$ describing the system of $N$ electrons in a spherical positive jellium into three parts [12]. The surface plasmon is represented by the centre-of-mass part $H_{\mathrm{cm}}=\hbar \tilde{\omega}_{\mathrm{M}} b^{\dagger} b$, where $b$ and $b^{\dagger}$ are the usual ladder operators of the harmonic oscillator. Its frequency $\tilde{\omega}_{\mathrm{M}}=\omega_{\mathrm{M}}\left(1-N_{\text {out }} / N\right)^{1 / 2}$ is reduced with respect to the bare Mie frequency $\omega_{\mathrm{M}}=\left(4 \pi n_{\mathrm{e}} e^{2} / 3 m_{\mathrm{e}}\right)^{1 / 2}$ due to the so-called spill-out effect which accounts for the fact that a fraction $N_{\text {out }} / N$ of the electrons is found outside the jellium sphere [13]. In the Mie frequency, $e, m_{\mathrm{e}}$, and $n_{\mathrm{e}}$ denote the charge, mass, and bulk density of the conduction electrons, respectively.

The relative coordinates constitute an environment leading to the decay of the plasmon. Within a mean-field approximation, one can write the corresponding part of the Hamiltonian as $H_{\text {rel }}=\sum_{\alpha} \varepsilon_{\alpha} c_{\alpha}^{\dagger} c_{\alpha}$, where the operators $c_{\alpha}^{\dagger}$ populate one-particle states with energies $\varepsilon_{\alpha}$ in the effective potential $V(r)$. These degrees of freedom are coupled to the plasmon by

$$
H_{\mathrm{c}}=\Lambda\left(b^{\dagger}+b\right) \sum_{\alpha \beta} d_{\alpha \beta} c_{\alpha}^{\dagger} c_{\beta},
$$

where $\Lambda=\left(\hbar m_{\mathrm{e}} \omega_{\mathrm{M}}^{3} / 2 N\right)^{1 / 2}$ and

$$
d_{\alpha \beta}=\left\langle\alpha\left|\left[z \Theta(a-r)+\frac{z a^{3}}{r^{3}} \Theta(r-a)\right]\right| \beta\right\rangle
$$

is a matrix element between two mean-field states. $\Theta(x)$ denotes the Heaviside step function.

Approximating the self-consistent mean-field potential by $V(r)=V_{0} \Theta(r-a)$, using semiclassical techniques (for details, see refs. $[8,11])$, and working up to second order in $T / T_{\mathrm{F}}$, we obtain the expansions (4) and (5) for the position of the plasmon resonance and its width, where

$$
\begin{gathered}
\frac{\omega_{\mathrm{sp}}^{(0)}}{\omega_{\mathrm{M}}}=1-\frac{3}{8 k_{\mathrm{F}} a} \xi_{0}\left(\frac{\varepsilon_{\mathrm{F}}}{\hbar \omega_{\mathrm{M}}}, \frac{\varepsilon_{\mathrm{F}}}{V_{0}}\right), \\
\frac{\omega_{\mathrm{sp}}^{(2)}}{\omega_{\mathrm{M}}}=\frac{3}{8 k_{\mathrm{F}} a} \xi_{2}\left(\frac{\varepsilon_{\mathrm{F}}}{\hbar \omega_{\mathrm{M}}}, \frac{\varepsilon_{\mathrm{F}}}{V_{0}}\right), \\
\gamma^{(i)}=\frac{3 v_{\mathrm{F}}}{4 a} g_{i}\left(\frac{\varepsilon_{\mathrm{F}}}{\hbar \omega_{\mathrm{M}}}\right), i=0,2 .
\end{gathered}
$$

Here, we have introduced the auxiliary functions $[8,11,14$, 15]

$$
\begin{array}{r}
\xi_{0}(x, y)=\frac{16 x^{3 / 2}}{15 \pi}\left[\ln \left(\frac{8 k_{\mathrm{F}} a}{3 \eta x g_{0}(x)}\right)-\frac{\pi}{2}-\frac{4}{3}\right] \\
+\frac{1}{y}[-\sqrt{y(1-y)}(2 y+3)+3 \arcsin \sqrt{y}], \\
\xi_{2}(x, y)=\frac{4 \pi}{9} x^{3 / 2}\left[\ln \left(\frac{8 k_{\mathrm{F}} a}{3 \eta x g_{0}(x)}\right)-\frac{\pi}{2}-\frac{4}{3}\right] \\
-\frac{16}{15 \pi} x^{3 / 2} \frac{g_{2}(x)}{g_{0}(x)}+\frac{\pi^{2}}{3}(2-y)\left(\frac{y}{1-y}\right)^{3 / 2}, \\
g_{0}(x)=\frac{1}{12 x^{2}}\{\sqrt{x(x+1)}(4 x(x+1)+3) \\
-3(2 x+1) \ln (\sqrt{x}+\sqrt{x+1}) \\
-\Theta(x-1)[\sqrt{x(x-1)}(4 x(x-1)+3) \\
-3(2 x-1) \ln (\sqrt{x}+\sqrt{x-1})]\}
\end{array}
$$




$$
\begin{array}{r}
g_{2}(x)=\frac{\pi^{2}}{24 x}\{\sqrt{x(x+1)}(6 x-1)+\ln (\sqrt{x}+\sqrt{x+1}) \\
-\Theta(x-1)[\sqrt{x(x-1)}(6 x+1) \\
+\ln (\sqrt{x}+\sqrt{x-1})]\} .
\end{array}
$$

$\varepsilon_{\mathrm{F}}, v_{\mathrm{F}}$, and $k_{\mathrm{F}}$ are the Fermi energy, velocity, and wavevector, respectively.

The dependence on $x$ of $\xi_{0}$ and $\xi_{2}$ arises from the coupling of the plasmon to the relative coordinates while the dependence on $y$ is due to the spill-out effect. Both effects depend quadratically on the temperature. The parameter $\eta$ is a cutoff of order unity that appears because the low-energy particle-hole excitations form the plasmon and thus do not contribute to its damping $[8,15]$. For numerical purposes we take $\eta=0.5$. It is important to remark that the finite temperature corrections scale as $1 / a$, and therefore become negligible for sufficiently large clusters. For a quantitatively correct description of very small nanoparticles $(a \lesssim 1 \mathrm{~nm})$, nonmonotonic size-dependent corrections have to be added [16]. In the case of noblemetal nanoparticles embedded in a glass matrix, the effect of the $d$ electrons and the dielectric mismatch at the border of the nanoparticle should be taken into account [17]. In the present context, the essential feature of the functions $g_{2}$ and $\xi_{2}$ is that they are positive. Therefore higher temperatures lead to a redshift of the resonance and to an increase of its width.

Numerical calculations within the temperaturedependent time-dependent local density approximation predicted a redshift of the resonance up to a systemdependent temperature, followed by a blueshift at higher temperatures [18]. This nonmonotonic feature has not been observed experimentally to our knowledge and is not captured by our low-temperature expansion. Systematic studies of the frequency shift and plasmon width have been carried out in gold [3] and silver [6] nanoparticles yielding a decrease of both corrections with increasing pump-probe time delay, i.e., decreasing electron temperature.

In fig. 11we used the analytic results presented in eqs. (4) and (5) for the temperature dependence of the plasmon resonance to describe the evolution of the differential transmission with decreasing electronic temperature. The curves present the qualitative features of the experimentally observed differential transmission of refs. [2] and [6]. As discussed after (6), the relaxation of the temperature leads to an anomaly at frequencies close to the plasmon resonance. We now investigate the properties of this anomaly in detail.

For different frequencies below (solid lines) and above (dashed lines) the frequency $\omega_{1}^{\mathrm{c}}$ we show in fig. 2 the relaxation dynamics normalized by the value at the maximum temperature $T_{\mathrm{M}}=0.05 T_{\mathrm{F}}$ reached after the excitation. An estimate for $T_{\mathrm{M}}$ can be obtained by assuming that the nanoparticle absorbs $n$ photons from the pump laser and increases its energy by about $n \hbar \omega_{\text {sp }}$. Using the low-temperature Sommerfeld expansion of the electronic energy one gets the temperature [11]

$$
\frac{T_{\mathrm{M}}}{T_{\mathrm{F}}} \simeq \sqrt{\frac{9 n \hbar \omega_{\mathrm{sp}}}{\pi \varepsilon_{\mathrm{F}}\left(k_{\mathrm{F}} a\right)^{3}}+\left(\frac{T_{0}}{T_{\mathrm{F}}}\right)^{2}} .
$$

For the sodium nanoparticle of fig. 1, one obtains for $n=$ $1(n=2)$ the temperature $T_{\mathrm{M}} / T_{\mathrm{F}} \approx 0.03(0.04)$. This corresponds to an electronic temperature of $T_{\mathrm{M}}=1100 \mathrm{~K}$ $(1600 \mathrm{~K})$.

The curves in fig. 2 can be related to the experimentally accessible decay rate

$$
\Gamma_{t}=-\frac{\mathrm{d}}{\mathrm{d} t} \ln \left(\frac{\Delta \mathcal{T}}{\mathcal{T}}\right) .
$$

In the case of an exponential relaxation, $\Gamma_{t}$ is independent of the time $t$. Since the differential transmission depends on time only implicitly through its dependence on temperature, the relaxation rate $\Gamma_{t}$ can be expressed as $\Gamma_{t}=-T \Gamma_{T} \mathrm{~d}(\ln T) / \mathrm{d} t$. The last factor on the righthand side corresponds to the relaxation rate of the electronic temperature and requires the precise knowledge of the time dependence $T(t)$ of the temperature. While it determines the order of magnitude of $\Gamma_{t}$, the dynamics of the relaxation process and in particular its frequency dependence is characterized by

$$
\Gamma_{T}=\frac{1}{\Delta \mathcal{T} / \mathcal{T}} \frac{\mathrm{d}}{\mathrm{d} T}\left(\frac{\Delta \mathcal{T}}{\mathcal{T}}\right)
$$

This rate is independent of the time dependence $T(t)$ of the temperature.

In the sequel of the paper, we will use $\Gamma_{T}$ taken at $T=T_{\mathrm{M}}$ in order to describe the relaxation process. This quantity is related to the slope of the differential transmission at $T_{\mathrm{M}}$, i.e., at the right end of the curves shown in fig. 22 The inverse of the relaxation parameter (14) is presented in the inset of fig. 2 as a function of the frequency.

For frequencies far away from the zeros of $\Delta \mathcal{T} / \mathcal{T}$, the relaxation parameter is almost frequency independent and $2\left(T_{\mathrm{M}} \Gamma_{T}\right)^{-1} \approx 1$. The factor of two arises since the differential transmission is proportional to $T^{2}$ in this regime. In contrast, a strong frequency dependence appears close to $\omega_{1}^{\mathrm{c}}$. For frequencies slightly below $\omega_{1}^{\mathrm{c}}$ the initial slope of the differential transmission is reduced and eventually even changes sign. Therefore the inverse relaxation parameter exhibits a divergence. The increase of the differential transmission with decreasing temperature for the uppermost dashed curve $\left(\omega / \omega_{\mathrm{sp}}^{(0)}=0.9849\right)$ can be traced back to the shift of $\omega_{1}^{\mathrm{c}}$ which overcompensates the amplitude relaxation for this value of $\omega$ (see the inset of fig. 1). For frequencies slightly above $\omega_{1}^{\mathrm{c}}$ we obtain very large slopes that decrease as we go further away from this frequency. As shown in the inset of fig. 2, between $\omega_{1}^{\mathrm{c}}$ and $\omega_{2}^{\mathrm{c}}$ a region appears where the relaxation parameter takes on values comparable to those far away from the resonance. While the anomaly around $\omega_{2}^{\mathrm{c}}$ resembles the one close to $\omega_{1}^{\mathrm{c}}$, its 


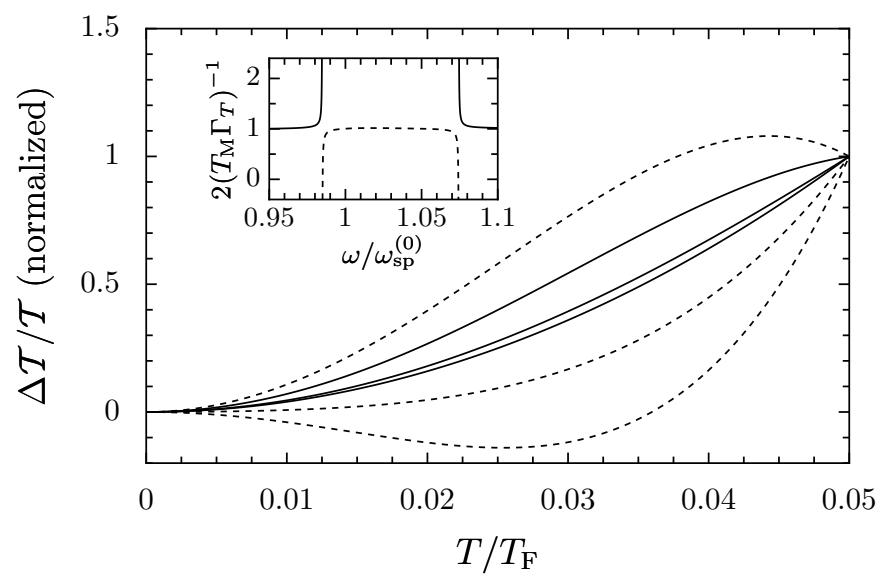

Fig. 2: The differential transmission normalised to the value at $T / T_{\mathrm{F}}=0.05$ is shown as a function of temperature. The system parameters are the same as in fig. 1. The solid lines correspond to the frequencies $\omega / \omega_{\mathrm{sp}}^{(0)}=0.9,0.983$ and 0.9847 from the lower to the upper curve while the dashed lines correspond to $\omega / \omega_{\mathrm{sp}}^{(0)}=0.9852,0.9854$ and 0.9849 from the lower to the upper curve. In the inset, the inverse of the relaxation parameter (14) is shown as a function of the frequency for $T_{\mathrm{M}} / T_{\mathrm{F}}=0.05$. The frequencies of the solid lines in the main graph lie on the left branch in the inset while the dashed lines lie on the middle branch.

quantitative description relies on less justified hypotheses like the absence of an interband transition close to $\omega_{2}^{\mathrm{c}}$ and the validity of the Breit-Wigner form over a broad frequency range.

In the experiment of ref. [2] on copper nanoparticles, a differential transmission lifetime was observed at $\omega / \omega_{\mathrm{sp}}^{(0)} \approx 0.99$ which is almost a factor of two larger than the lifetimes found at $\omega / \omega_{\mathrm{sp}}^{(0)} \approx 0.97$ and 1.025 . Using the initial electronic temperature estimated to be about $800 \mathrm{~K}[2]$ and the parameters of the experiment $k_{\mathrm{F}} a=68$ and $\varepsilon_{\mathrm{F}} / \hbar \omega_{\mathrm{sp}}=3.2$, we find that the frequency at which the lifetime is a factor of two larger than far from the anomaly reproduces quantitatively the experimentally observed value. In addition, the increase of the lifetime is present only inside a very narrow frequency range whose width is smaller than the separations of the experimental points. Thus, in agreement with the experimental findings, a variation of the frequency by only $2 \%$ can yield an enlargement of the differential transmission lifetime by a factor of two.

In fig. 3 we present $\Gamma_{T}^{-1}$ for the sodium nanoparticle of figs. 1 and 2 as a function of the initial temperature $T_{\mathrm{M}}$ for various frequencies around $\omega_{1}^{\mathrm{c}}\left(T_{\mathrm{M}}\right)$. Consistently with our findings for the frequency dependence of $\Gamma_{T}$, we find for frequencies far from $\omega_{1}^{\mathrm{c}}$ (cf. the two lower curves) an almost frequency-independent relaxation parameter which only weakly increases with the maximum temperature $T_{\mathrm{M}}$. On the other hand, for frequencies just below $\omega_{1}^{\mathrm{c}}$ (cf. the uppermost curve), we see that $\Gamma_{T}^{-1}$ is rapidly increasing with $T_{\mathrm{M}}$ beyond $T_{\mathrm{M}} / T_{F}=0.03$. For higher initial tem-

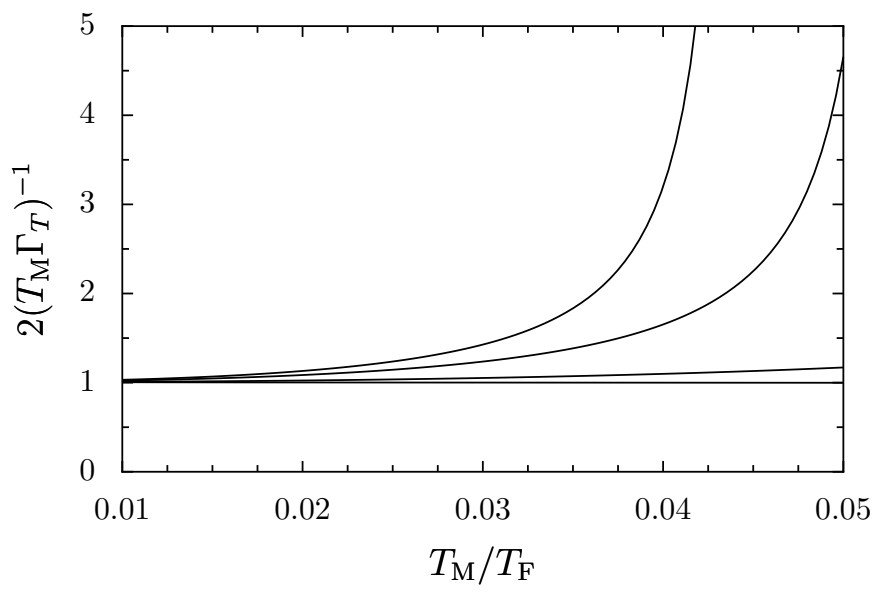

Fig. 3: The inverse relaxation parameter (14) for the same system parameters as in fig. 1 is shown as a function of the maximum electronic temperature $T_{\mathrm{M}}$ in the nanoparticle. The frequencies are $\omega / \omega_{\mathrm{sp}}^{(0)}=0.9,0.983,0.9847$, and 0.9849 from the lower to the upper curve.

peratures $T_{\mathrm{M}}, \Gamma_{T}^{-1}$ will eventually become negative as discussed above.

The results presented in fig. 3 can be compared with experiments because the initial temperature $T_{\mathrm{M}}$ is related to the experimentally controlled pumping energy density which determines the energy initially deposited in a nanoparticle (see eq. (12)). By measuring the relaxation rate on and off resonance, it was found in ref. [4] that at an estimated average flux of one pump photon per nanoparticle a transition from a frequency-independent to a frequency-dependent relaxation rate occurs. Above this threshold, the inverse relaxation rate on resonance is significantly larger than off resonance. This scenario is clearly reproduced by the theoretical results shown in fig. 3. Consistently with the experimental findings, the lifetime can be increased by a factor of two when the number of absorbed photons is larger than one, i.e., for $T_{\mathrm{M}} / T_{\mathrm{F}}>0.03$.

While the slowdown of the transmission dynamics has been measured, an acceleration has not yet been reported in the literature. However, extracting the frequencydependent relaxation times close to the resonance is quite difficult because there the initial value of $\Delta \mathcal{T} / \mathcal{T}$ used to normalise the data is very small. Systematic experiments measuring the differential transmission relaxation as a function of frequency would nevertheless be highly desirable in order to confirm unambiguously that the mechanism we propose is at work.

In summary, we have shown that the relaxation dynamics of the differential transmission of a nanoparticle exhibits an anomaly close to the surface plasmon resonance. This anomaly is due to the temperature dependencies of the position and the width of the surface plasmon resonance and manifests itself as a slowdown for frequencies below the plasmon frequency. In addition, in a narrow 
frequency window we find a faster relaxation.

The slowdown of the relaxation of the differential transmission close to the plasmon resonance has been observed experimentally for copper and silver nanoparticles $[2,4,5]$, and several of the key findings are reproduced by our approach, including the fact that the anomaly disappears under weak excitations or for relatively large clusters.

The previously proposed explanation of the anomaly as a size-dependent many-body effect based on the resonant scattering of the $d$ holes into the conduction band [7] cannot be invoked in the absence of interband transitions close to the surface plasmon resonance, as it is the case for silver nanoparticles. In contrast, the mechanism presented here does not rely on interband transitions and applies to a large variety of metallic nanoparticles. This demonstrates that the interband transition is not essential for the appearance of the relaxation anomaly. In particular, it leads us to predict that a considerable slowdown of the transmission relaxation should also be observable in alkaline nanoparticles.

Our explanation does not invoke any special behaviour of the electron dynamics at the plasmon frequency. In our view, the relaxation of the electronic temperature fully describes the cooling of the electron gas on timescales which are larger than the lifetime of the surface plasmon. The time evolution of this temperature may depend on physical parameters, like the size of the nanoparticle $[6,19]$ or the medium in which it is embedded [20], but the decreasing electronic temperature is not expected to be influenced by the frequency at which the optical transmission is detected. The anomaly with a strong frequency dependence of the relaxation rate appears only when the differential optical transmission is considered.

$$
* * *
$$

We thank J.-Y. Bigot, V. Halté and P.-A. Hervieux for stimulating discussions. RAJ benefited from the hospitality of the Institut für Physik der Universität Augsburg during the final phase of this work. Financial support through the French-German PAI program Procope and from the European Union through the MCRTN program is gratefully acknowledged.

\section{REFERENCES}

[1] Tokizaki T. et al., Appl. Phys. Lett., 65 (1994) 941.

[2] Bigot J.-Y., Merle J.-C., Cregut O. and Daunois A., Phys. Rev. Lett., 75 (1995) 4702.

[3] Perner M. et al., Phys. Rev. Lett., 78 (1997) 2192.

[4] Halté V. et al., Phys. Rev. B, 60 (1999) 11738.

[5] Bigot J.-Y., Halté V., Merle J.-C. and Daunois A., Chem. Phys., 251 (2000) 181.

[6] Del Fatti N. et al., Chem. Phys., 251 (2000) 215; Voisin C., Del Fatti N., Christofilos D. and Vallée F., J. Phys. Chem. B, 105 (2001) 2264.
[7] Shahbazyan T. V., Perakis I. E. and Bigot J.-Y., Phys. Rev. Lett., 81 (1998) 3120; Shahbazyan T. V. and Perakis I. E., Phys. Rev. B, 60 (1999) 9090.

[8] Weick G., Ingold G.-L., Jalabert R. A. and WeinMANn D., Phys. Rev. B, 74 (2006) 165421.

[9] Hervieux P.-A., Benabbas A., Halté V. and Bigot J.-Y., Eur. Phys. J. D, 24 (2003) 185.

[10] Eesley G. L., Phys. Rev. B, 33 (1986) 2144.

[11] Weick G., Ph.D. Thesis, Université Louis Pasteur Strasbourg and Universität Augsburg (2006), URL: http://tel.ccsd.cnrs.fr/tel-00103438.

[12] Gerchikov L. G., Guet C. and Ipatov A. N., Phys. Rev. A, 66 (2002) 053202.

[13] de Heer W. A., Rev. Mod. Phys., 65 (1993) 611; Brack M., ibid., 65 (1993) 677.

[14] Barma M. and Subrahmanyam V., J. Phys.: Condens. Matter, 1 (1989) 7681.

[15] Yannouleas C. and Broglia R. A., Ann. Phys. (N. Y.), 217 (1992) 105.

[16] Molina R. A., Weinmann D. and Jalabert R. A., Phys. Rev. B, 65 (2002) 155427.

[17] Weick G., Molina R. A., Weinmann D. and Jalabert R. A., Phys. Rev. B, 72 (2005) 115410.

[18] Hervieux P.-A. and Bigot J.-Y., Phys. Rev. Lett., 92 (2004) 197402.

[19] Darugar Q., Qian W., El-Sayed M. A. and Pileni M.-P., J. Phys. Chem. B, 110 (2006) 143.

[20] Link S. and El-Sayed M. A., J. Phys. Chem. B, 103 (1999) 8410. 